\title{
Limb-saving Knee Arthrodesis with a Silver-coated Arthrodesis Rod in a Patient with Aspergillus Osteomyelitis of the Knee
}

\author{
Abdullah Ismat ${ }^{1}$, Volker Alt ${ }^{2}$, Christian Heiss $^{3}$, Markus Rupp $^{4}$
}

\begin{abstract}
Aspergillus osteomyelitis can be a very debilitating disease with an associated mortality of up to $25 \%$. Silver is well known for its broad antimicrobial properties including activity against fungi and the coating of orthopaedic implants with silver has been reported to be successful in complex cases. We report for the first time on a complicated case of Aspergillus osteomyelitis affecting the left knee in a patient with a chronic lymphoid leukaemia treated with appropriate surgical debridement, local and systemic antibiotic and antimycotic therapy, as well as staged surgical treatment with final limb-saving arthrodesis by using a custom-made microsilver coated arthrodesis rod.

Keywords: Antifungal agents, Arthrodesis, Aspergillosis, Fungal osteomyelitis, Silver-coated.

Strategies in Trauma and Limb Reconstruction (2020): 10.5005/jp-journals-10080-1445
\end{abstract}

\section{INTRODUCTION}

Infection with Aspergillus species in humans can be of variable severity, ranging from allergies and disease primarily affecting the lungs to invasive aspergillosis and dissemination. Aspergillus fumigatus has been shown to be the most prevalent fungal pathogen to humans. ${ }^{1}$ The spread of disease is known to occur mainly through airborne transmission. ${ }^{2}$ As with any infectious disease, host factors particularly the immune status play a determining role in the extent of disease. ${ }^{3}$ There has been an increase in the prevalence of invasive aspergillosis correlated to the improving rate of survival resulting from more aggressive treatment regimens applied in different hemato-oncological conditions. ${ }^{4}$ However, mortality of patients suffering from Aspergillus osteomyelitis is reported to be up to $25 \%{ }^{5}$ Therefore, limb-saving surgical treatment options in seriously ill patients is not the primary treatment goal, but possible. Standardized treatment regimens for fungal infections, particularly involving surgical intervention, are generally lacking. Treatment needs to be based on an individual basis relating not only to the patient but also the treating surgeon's experience. On this background, the authors hereby report for the first time on a complex case of Aspergillus osteomyelitis affecting the left knee joint in a patient with a chronic lymphoid leukaemia, finally treated with knee arthrodesis by using a silver-coated, antimycotic arthrodesis rod.

\section{Case Description}

The patient is a 67-year-old male who was initially referred to our trauma and orthopaedic services from the haematology department with a one-month history of an atraumatic hematoma of the left knee presenting with increasing pain and swelling. A background history of chronic lymphoid leukaemia (CLL), that was diagnosed 15 years ago, was present. The patient was started on ibrutinib $420 \mathrm{mg} /$ day 6 months beforehand for his CLL and the hematoma was assumed to have occurred as a side effect from this medication. On examination, the knee was swollen with a livid discoloration and the knee joint was warm to

\begin{abstract}
${ }^{1-4}$ Department of Trauma, Hand and Reconstructive Surgery, University Hospital Giessen-Marburg GmbH, Giessen, Germany

Corresponding Author: Abdullah Ismat, Department of Trauma, Hand and Reconstructive Surgery, University Hospital GiessenMarburg GmbH, Giessen, Germany, Phone: +49 64198544601, e-mail: Abdullahismat0@gmail.com

How to cite this article: Ismat A, Alt V, Heiss C, et al. Limb-saving Knee Arthrodesis with a Silver-coated Arthrodesis Rod in a Patient with Aspergillus Osteomyelitis of the Knee. Strategies Trauma Limb Reconstr 2020;15(2):121-125.
\end{abstract}

Source of support: Nil

Conflict of interest: None

touch with markedly limited extension and flexion. After initial conservative treatment by his GP, the symptoms persisted and continued to worsen. Hence, magnetic resonance imaging (MRI) was then performed. It demonstrated arthritis and osteomyelitis of the knee. Additional computer tomography (CT) could rule out sequestra and osseous fistula. Initial antibiotic therapy with piperacillin/tazobactam was initiated as the inflammatory markers were elevated. The patient was operated on the knee and an infected hematoma of the knee joint was evident intraoperatively. Further surgical interventions with vacuumassisted closure (VAC) therapy and subsequent changing of the VAC dressing every 3-5 days were performed.

Cultures provided evidence of Aspergillus fumigatus and treatment with the antimycotic agent posaconazole was commenced. Subsequently, a positron emission tomographycomputed tomography (PET-CT) demonstrated infection in the left knee extending to the lower leg without other foci of infection (Fig. 1). After evidence of Staphylococcus epidermidis and antibiotic sensitivity testing, the antibiotic therapy was changed to fosfomycin, vancomycin, and imipenem accordingly.

The wound was then closed secondarily a month after the initial surgery following adequate conditioning through the VAC therapy and decontamination. 
After discharge of the patient, the antimycotic agent posaconazole administered orally with a dosage of $300 \mathrm{mg}$ once daily was to be continued for 6 weeks. After 9 months, the patient underwent an arthroscopy of the knee as an MRI investigating recurrent symptoms demonstrated evidence of osteomyelitis and arthritis of the left knee joint with involvement of the femur, tibia, and patella (Fig. 2). After primary arthroscopic joint irrigation and partial synovectomy of the knee, several revision surgeries were necessary to ensure decontamination of the infected joint. The patient was then thoroughly consulted about the need of surgical treatment by means of radical debridement and resection of infected bone around the knee, which would finally result in an arthrodesis of the knee joint. A two-stage procedure with precedent placement of an antibiotic and antimycotic spacer was planned. Surgery was performed with insertion of PALACOS ${ }^{\circledR}$ bone cement (Heraeus, Wehrheim, Germany) with caspofungin, Cancidas ${ }^{\oplus}$ (MSD; Kenilworth, NJ, USA) $70 \mathrm{mg}$, and a $4.5-\mathrm{mm}$ titanium rod $(6 \times 110$ $\mathrm{mm}$ ) (Fig. 3). The titanium rods were coated with bone cement in the silicone tubing technique as described by Thonse et al. in 2008. Multiple further revision surgeries were performed during this admission. Aspergillus fumigatus was evident from intraoperative

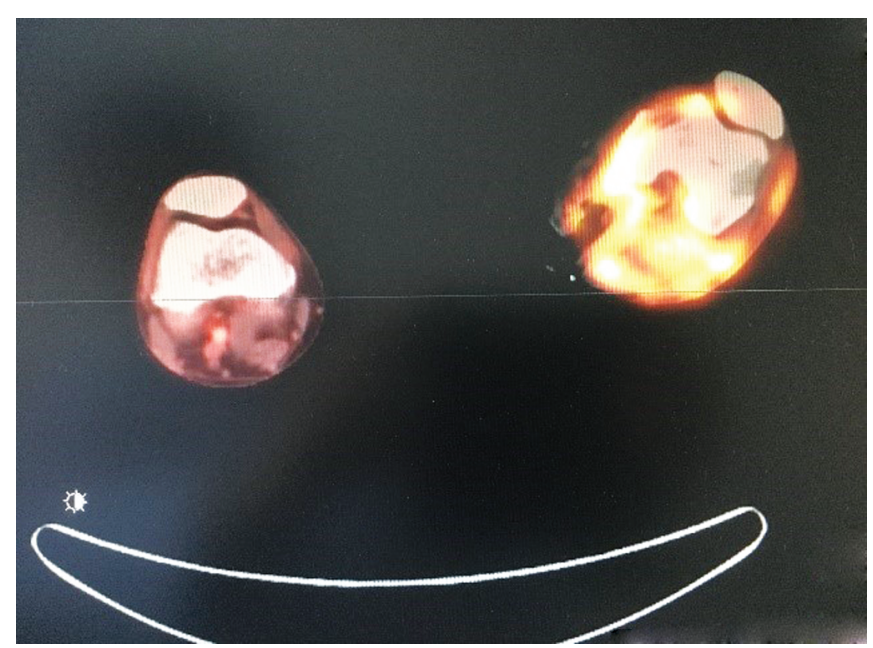

Fig. 1: Positron emission tomography-computed tomography finding: Extensively increased fludeoxyglucose (FDG) uptake in the left distal upper leg, knee with involvement of the joint capsule, and proximal lower leg. Further localization in the left gastrocnemius and tibialis anterior musculature tissue samples obtained during one of the revision surgeries and oral voriconazole (antimycotic agent) was given in addition to the locally applied Cancidas ${ }^{\circledR}$ cement until discharge. Moreover, intravenous vancomycin (antibiotic agent) was also provided as Staphylococcus haemolyticus was evident in the intraoperative samples from the primary surgery.

Three weeks after discharge, increasing pain in the left knee radiating to the left lower leg was reported by the patient and a moderate effusion of the knee with increased warmth and redness was evident on examination. The knee was aspirated on admission and had to be aspirated again twice due to persistent hematoma formation. The microbiological examination of the puncture specimens demonstrated evidence of Aspergillus fumigatus. A revision surgery with further bone resection of the distal femur was necessary. Due to recurrence of infection with need of revision surgery, local antibiotic and antimycotic therapy was changed. $300 \mathrm{mg}$ liposomal amphotericin B was admixed to a $80 \mathrm{~g} \mathrm{Copal}{ }^{\oplus}$ $\mathrm{G}+\mathrm{V}$ cement spacer (Copal ${ }^{\circledR} \mathrm{G}+\mathrm{V}$, Haereus, Wehrheim, Germany); 40 $\mathrm{g}$ poly (methyl methacrylate) (PMMA) containing additional $0.5 \mathrm{~g}$ of gentamicin and $2 \mathrm{~g}$ of vancomycin as supplementing local antibiotic agents (Fig. 4). The systemic antimycotic therapy was then followed up with voriconazole and amphotericin B. The patient's general condition deteriorated postoperatively, probably due to evolving sepsis from the Aspergillus infection. Blood cultures demonstrated evidence of Staphylococcus epidermidis and Enterococcus faecium. An evident immunoglobulin deficiency was treated with intravenous immune globulin (Privigen ${ }^{\circledR}$ ) and an antibiotic therapy with piperacillin and tazobactam (antibiotic agent) was also provided. A PET-CT demonstrated a regressive pneumonia with known infiltration of all lymph nodes as part of the CLL.

Admission for arthrodesis of the left knee joint with an individually customized rod took place 2 weeks after the most recent discharge. Infect eradication was evident by a negative tissue sample obtained by preoperative biopsy as well as a laboratory test of the C-reactive protein level, which normalized in the meantime $(<0.5 \mathrm{mg} / \mathrm{dL})$. An arthrodesis rod consisting of a femoral and a tibial nail measuring $200 \mathrm{~mm}$ long each with a diameter of 18 and $14 \mathrm{~mm}$, respectively, connected with a central 125-mm-long spacer with a diameter of $40 \mathrm{~mm}$ (OsteoBridge ${ }^{\mathrm{Tm}}$, Merete $\mathrm{GmbH}$, Berlin, Germany) was coated with microsilver (HyProtect ${ }^{\mathrm{TM}}$, BioGate AG, Nuremberg, Germany) as a local strategy to reduce the postoperative infection risk. The silver content of the $90-\mathrm{mm}$-thick coating was $2.7 \mu \mathrm{g} / \mathrm{cm}^{2}$ with a total silver content of the implant
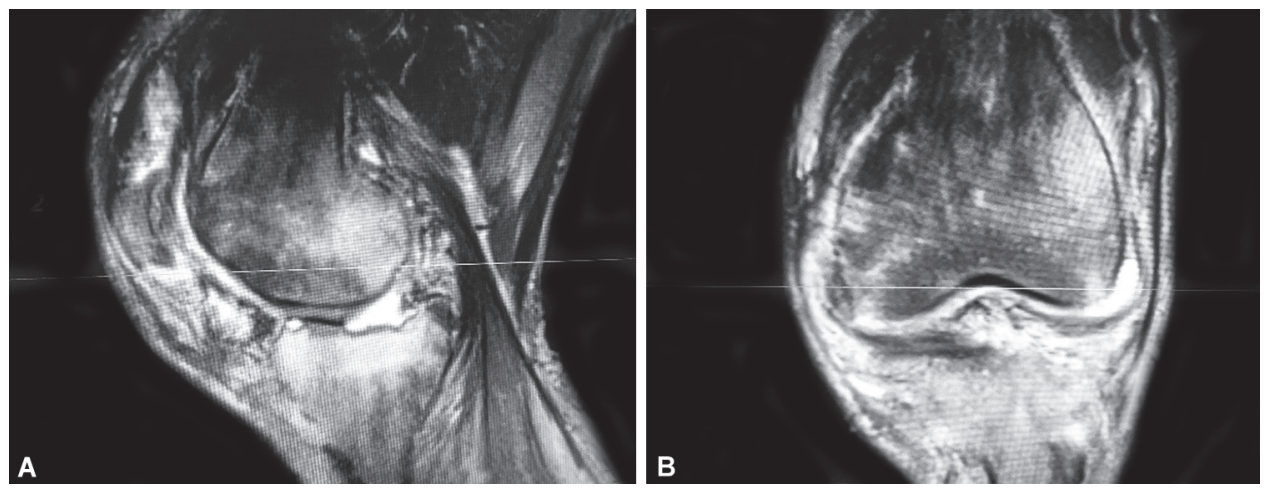

Figs 2A and B: Magnetic resonance imaging [T1-weighted image with fat suppression sagittal and coronal planes and T1-FS (fat suppressed) post contrast] demonstrates osteomyelitis and arthritis of the left knee with femoral, tibial, and patellar involvement. Advanced destruction of the cartilage and subchondral bone at the tibiofemoral and femoropatellar joints medially and laterally. Additional subtotal destruction of the menisci and inflammation of the patellar ligament close to its insertion point. The collateral and cruciate ligaments appear intact 

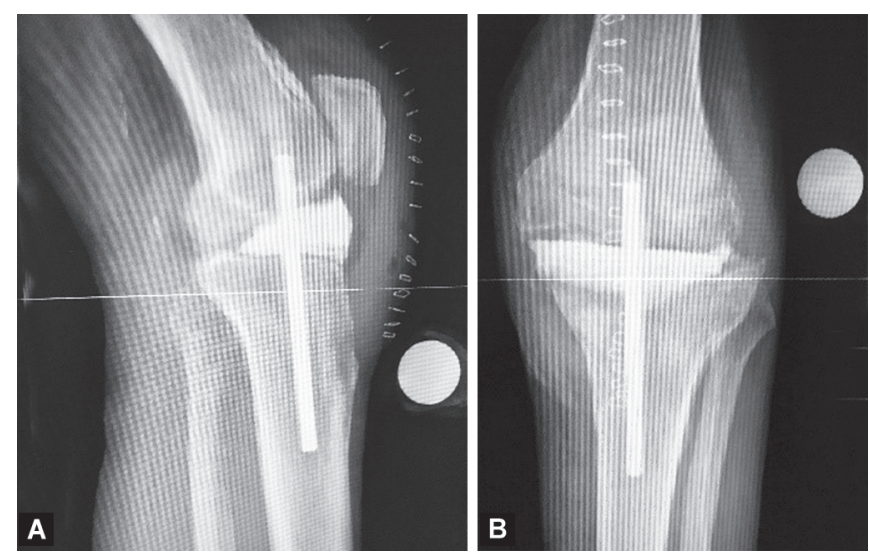

Figs $3 \mathrm{~A}$ and B: Postoperative X-rays (after debridement and total synovectomy). Bone drilling of the distal femur and proximal tibia with femoral shortening osteotomy. Insertion of PALACOS ${ }^{\circledR}$ bone cement with Cancidas ${ }^{\circledast} 70 \mathrm{mg}$ and a Xia titanium rod $(6 \times 110 \mathrm{~mm})$

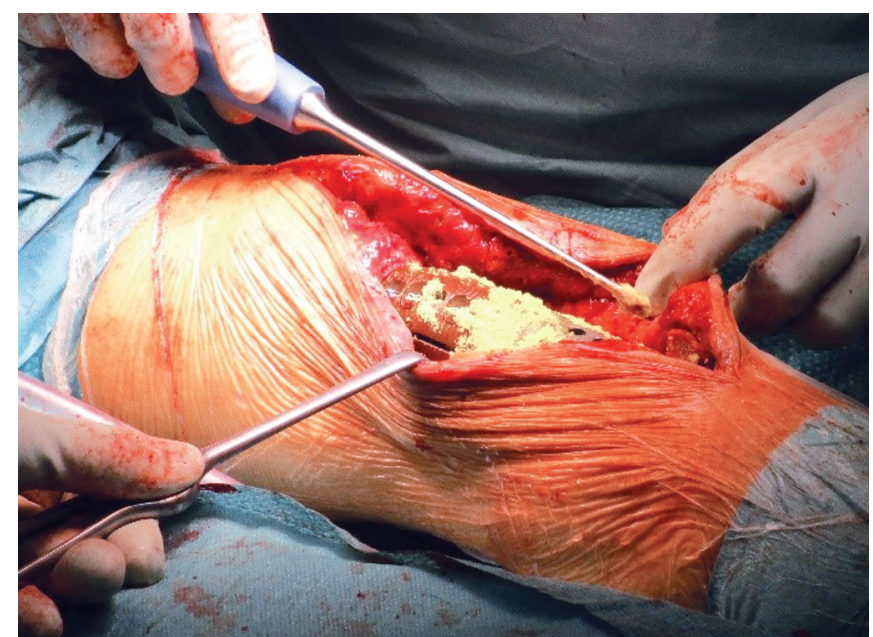

Fig. 5: Intraoperative image outlining local application of $150 \mathrm{mg}$ amphotericin B around the implanted microsilver-coated arthrodesis rod

of around $1064 \mu \mathrm{g}$. This silver-coated arthrodesis implant was then inserted after another debridement with additional local application of $150 \mathrm{mg}$ amphotericin B around the microsilver-coated arthrodesis rod before closure (Fig. 5). Voriconazole (antimycotic agent) was additionally administered orally. Granocyte ${ }^{\oplus}$ (33.6 million IU) was also given every 2 days. Microbiological examination of the intraoperative tissue samples continued to demonstrate no further evidence of infection. The patient was then discharged as the inflammatory markers continued to normalize and the patient's condition improved. The patient was able to weight-bear on the affected knee and was mobilizing with crutches on discharge.

The patient was admitted after 2 weeks to the hematology services and was started back on a course of chemotherapy with the agent venetoclax after completed treatment of the Aspergillus osteomyelitis and appropriate postoperative healing. The patient continued to report no further symptoms or pain in the left knee. The knee was slightly swollen and warm 3 weeks postoperatively but non-tender. The patient was able to mobilize and partially weight-bear on the affected knee using crutches without any pain.

The patient was then regularly followed up in our outpatient clinic at 2 and 4 weekly intervals for more than 12 months

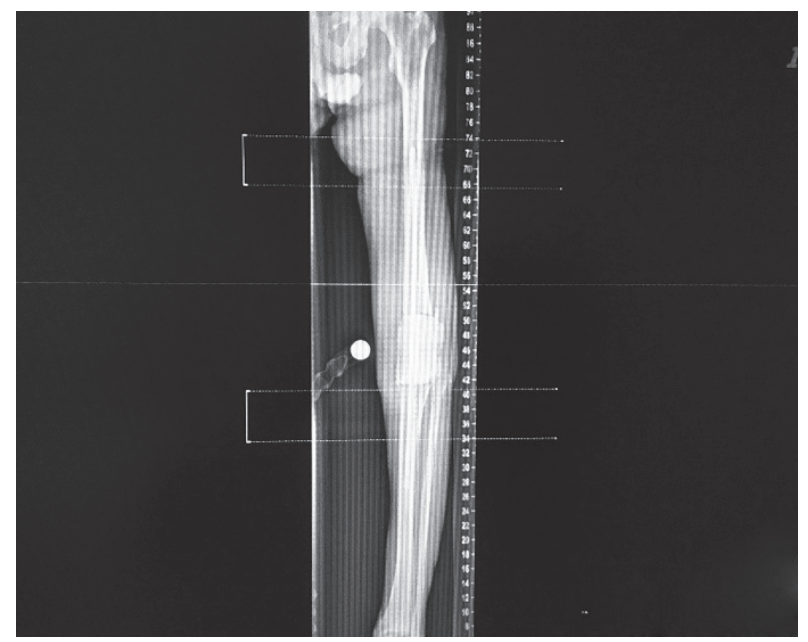

Fig. 4: After spacer and rod exchange and further resection of the distal femur
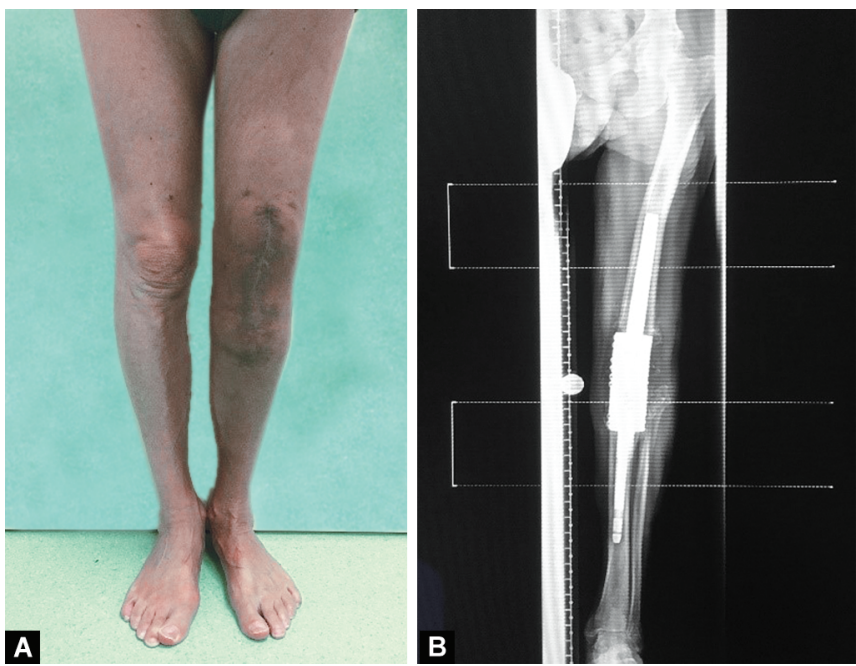

Figs $6 \mathrm{~A}$ and B: Follow-up 12 months after final surgery with microsilvercoated customized arthrodesis: well-healed surgical scar. Non-tender on palpation, with no increased warmth or redness. Weight-bearing on the affected limb possible and nonpainful

postoperatively and subsequently at yearly intervals for a total follow-up period of 30 months to date. He continued to report no symptoms in the left knee. On clinical examination, the knee was initially swollen but non-tender. On further follow-up, the knee swelling was regressive in nature, and no redness or increased warmth was evident. Full weight-bearing was possible without any pain. The CRP level continued to remain unremarkable and X-ray continued to demonstrate proper placement of the nail arthrodesis and no further radiological abnormalities (Fig. 6). Furthermore, no silver-specific adverse events such as argyria were found over the 30-month follow-up period so far.

\section{Discussion}

As illustrated by the demonstrated case, even in immunocompromised patients suffering from chronic Aspergillus osteomyelitis, limb-saving treatment is possible. To the best of our knowledge, there has not been any work to date reporting on the clinical 
application and antifungal properties of silver acting specifically against Aspergillus species in the setting of osteomyelitis. In our patient, definitive surgical intervention with use of silver coating aiding local and systemic antimycotic therapy has achieved remission of infection and has prevented recurrence during the period of follow-up.

Fungal arthritis and osteomyelitis are usually caused by Candida species. ${ }^{6}$ However, when Aspergillus species cause bone infection, they mostly affect the vertebrae, ribs, and cranial bones. ${ }^{7}$ Aspergillus osteomyelitis affects long bones in just $9 \%$ of cases. ${ }^{5}$ Haematogenous spread is the most common reason for Aspergillus species-caused bone infection (44\%), followed by contiguous infection (32\%), and direct inoculation (23\%), which may result after open fractures and surgery. ${ }^{7}$ In our immunocompromised patient suffering from CLL, pulmonary aspergillosis as the primary focus is likely as PET-CT showed evidence of pneumonia over the course of treatment. The treatment was complicated by a mixed bacterial fungal infection. Besides Aspergillus fumigatus, cultures of intraoperative tissue samples revealed bacterial superinfection with Staphylococcus epidermidis first and then Staphylococcus hemolyticus further complicating the treatment. Reasons may be intraoperative contamination, VAC therapy, or septicemia due to biofilm formation on catheters, which were placed into the patient over the course of the treatment. Polymicrobial infections cause osteomyelitis in up to $29 \% .{ }^{8}$ However, mixed fungal and bacterial infections are rare. To date, only one other case of combined fungal and bacterial osteomyelitis has been reported in the literature. ${ }^{9}$ $A$ reason for this could be that fungal cultures and polymerase chain reaction (PCR)-based diagnosis are not routinely performed in microbiological diagnostics. ${ }^{10}$ To analyse the protracted and complex course in our case, diagnosis of osteomyelitis has to be reviewed. Clinical examination is a cornerstone for the diagnosis of osteomyelitis. However, due to unspecific signs and symptoms in osteomyelitis additional radiological diagnostics are indispensable. Early diagnosis may prevent surgical therapy, which is necessary after development of chronic osteomyelitis. As the initial signs and symptoms in the patient were not immediately indicative of osteomyelitis, we performed basic diagnostics including conventional X-rays and MRI (Fig. 2). Both diagnostic modalities are widely spread and available nowadays and present appropriate radiological diagnostic methods of osteomyelitis. ${ }^{11}$ Additional CT was performed to rule out presence of sequestra as a sign of chronic osteomyelitis, which would require immediate surgical debridement and bone resection. Benefit of additional surgery together with extended antimycotic therapy for fungal bone infections is still under discussion. Gabrielli could not show better results for additional surgical treatment, ${ }^{5}$ whereas Gamaletsou ${ }^{7}$ and Koehler ${ }^{12}$ could show better survival rates in patients suffering from fungal osteomyelitis treated by antifungal therapy with additional surgery. Gabrielli referred to 310 patients treated between 1936 and 2013. Changes in indications, surgical techniques, and antifungal medication over time may be reason for the differing results. Nevertheless, we performed arthroscopic lavage and soft tissue debridement to reduce the number of organisms in addition to systemic antimycotic and antibacterial treatment. Both, antimycotic and antibiotic therapy were carried out according to the culture results and susceptibility testing. As long as no clear guidelines for the antimycotic treatment duration exist, clinical practice obliges surgeons to base antimycotic treatment on experience with bacterial infections. Five weeks of antimycotic in-hospital treatment was extended to additional 6 weeks of oral posaconazole after discharge of the patient. Thus, the widely but empirically used 6 weeks of antibiotic therapy in bacterial osteomyelitis have been considerably exceeded. ${ }^{13}$ Although, surgery as well as medical antimicrobial therapy were carried out in our case, osteomyelitis reappeared again after 9 months. After this period, chronic osteomyelitis had to be assumed. Accompanying biofilm formation, either of bacteria or fungi, complicates the treatment since most of antibiotics and antimycotics are not effective against biofilm and microbes in their sessile phase. ${ }^{14}$ Therefore, surgery with radical debridement and resection of the affected bone was necessary to achieve infection eradication. Local as well as systemic antibiotic and antimycotic therapy were administered. In general, amphotericin B, triazoles, and echinocandins are all antifungal drugs, which could be used for Aspergillus spp. infections. Since no superiority of any pharmacologic antifungal agent could be demonstrated, ${ }^{5,7}$ specific features of the antimycotic drugs must be taken into account for treatment decision. Drawbacks of amphotericin B are cumulative nephrotoxicity and the need for intravenous application with accompanying morbidity of prolonged venous access. Voriconazole has the flexibility of parenteral and oral formulations. Echinocandins act by noncompetitive inhibition of 1,3-b-glucan, which is a unique effector mechanism. This allows combination of echinocandins with other antifungals. Because caspofungin and micafungin seem to be effective against fungal biofilms, echinocandins may be considered in case of hardware retention. ${ }^{6}$ Needless to say, side effects and toxicities have to be considered in treatment decisionmaking, especially in severely ill patients. To avoid undesired side effects, local application of antifungal therapy is a sound treatment option. Consistent elution levels of amphotericin B were reported for more than 110 days. ${ }^{15}$ For voriconazole, sufficient elution could be demonstrated for 2 weeks only. ${ }^{16}$ Caspofungin has not been examined for its elution characteristics out of PMMA. However, other echinocandins like micafungin and anidulafungin appear to be poor candidates for local delivery into bone cement. ${ }^{15,17}$ In the presented case, caspofungin was added to the initial PMMA spacer because of its biofilm activity. Later on, additional revision surgery was necessary and amphotericin B was added to the PMMA spacer due to its favourable elution kinetics.

After eradication of infection, successful arthrodesis was carried out by a custom-made silver-coated arthrodesis rod. In this challenging case, with the patient at high risk of recurrent fungal infection and bacterial superinfection, best possible infection prevention was necessary. Because of the large foreign surface of the arthrodesis rod, we decided to use a silver-coated implant. As a reduction of prosthetic joint infections could be demonstrated in silver-coated tumor megaprothesis. ${ }^{18,19}$ In vitro, microsilver has been shown to act against gram-positive, negative, and even multi-resistant strains, without toxic side effects. ${ }^{20}$ Furthermore, silver nanoparticles have been demonstrated to poses antifungal activity against Candida species ${ }^{21}$ and Aspergillus fumigatus. ${ }^{22}$ We chose a $\mathrm{Ag} / \mathrm{SiO}_{x} \mathrm{C}_{y}$ plasma polymer coating with microsilver to reduce the risk of local toxicity. ${ }^{23}$ The total amount of silver in a Ag/SiOxCy plasma polymer coating is considerably less than in galvanic silver-coated implants, ${ }^{23}$ in which local agyria was reported after tumour prosthesis implantation. ${ }^{24}$ Although being off-label use, we administered $150 \mathrm{mg}$ amphotericin $\mathrm{B}$ around the silver-coated arthrodesis rod as an additional antimycotic measure. This unusual action was based on in vitro findings, which could demonstrate a synergistic antifungal effect of silver 
nanoparticles with amphotericin B. ${ }^{25}$ No negative side effects have been determined after application of a double daily intravenous dose (150 mg amphotericin B) for a 75-kg patient, which shows that additional local administration of amphotericin $B$ is feasible. Moreover, no silver-associated adverse event such as local argyria was found confirming the good biocompatibility of the used microsilver coating technology.

In summary, appropriate surgical debridement, local and systemic antibiotics/antimycotics, medical optimization of the immunocompromised patient, and modern antimicrobial coating with final limb-saving arthrodesis by using a custom-made microsilver-coated arthrodesis rod have all contributed to successful treatment with no recurrence of infection so far.

\section{Conclusion}

Aspergillus osteomyelitis is a very debilitating disease especially in immunocompromised patients. To avoid chronic osteomyelitis, early diagnosis and treatment are necessary. In chronic osteomyelitis, local and systemic antifungal treatment combined with appropriate surgical debridement is necessary to achieve eradication of infection. Silver coating applied to implants used in these cases may augment operative treatment, as demonstrated in this case.

\section{Ethical Statement}

The patient's informed consent was obtained for the purposes of this publication.

\section{Author Contributions}

Abdullah Ismat collected data, designed, drafted, and critically revised the manuscript. Volker Alt and Markus Rupp reviewed and critically revised the manuscript. Christian Heiss provided administrative, technical, and supervisory support. All authors read and approved the final manuscript.

\section{References}

1. Heinekamp T, Schmidt H, Lapp K, et al., Interference of Aspergillus fumigatus with the immune response. Paper presented at: Seminars in immunopathology 2015.

2. Fernstrom A, Goldblatt M. Aerobiology and its role in the transmission of infectious diseases. Journal of pathogens 2013;2013.

3. Geiser DM. Sexual structures in Aspergillus: morphology, importance and genomics. Med Mycol 2009;47(Suppl 1):S21-S26. DOI: 10.1080/13693780802139859.

4. Maschmeyer G, Haas A, Cornely OA. Invasive aspergillosis. Drugs 2007;67(11):1567-1601. DOI: 10.2165/00003495-200767110-00004.

5. Gabrielli E, Fothergill AW, Brescini L, et al. Osteomyelitis caused by Aspergillus species: a review of 310 reported cases. Clin Microbiol Infect 2014;20(6):559-565. DOI: 10.1111/1469-0691.12389.

6. Bariteau JT, Waryasz GR, McDonnell M, et al. Fungal osteomyelitis and septic arthritis. J Am Acad Orthop Surg 2014;22(6):390-401. DOI: 10.5435/JAAOS-22-06-390.

7. Gamaletsou MN, Rammaert B, Bueno MA, et al. Aspergillus osteomyelitis: epidemiology, clinical manifestations, management, and outcome. J Infect 2014;68(5):478-493. DOI: 10.1016/ j.jinf.2013.12.008.
8. Sheehy SH, Atkins BA, Bejon P, et al. The microbiology of chronic osteomyelitis: prevalence of resistance to common empirical anti-microbial regimens. J Infect 2010;60(5):338-343. DOI: 10.1016/ j.jinf.2010.03.006.

9. Giger A, YusufE, Manuel O, et al. Polymicrobial vertebral osteomyelitis after oesophageal biopsy: a case report. BMC Infect Dis 2016;16(1):141. DOI: 10.1186/s12879-016-1471-9.

10. Patterson TF, Thompson GR, Denning DW, et al. Practice guidelines for the diagnosis and management of aspergillosis: 2016 update by the infectious diseases society of America. Clin Infect Dis 2016;63(4): e1-e60. DOI: 10.1093/cid/ciw326.

11. Lew DP, Waldvogel FA. Osteomyelitis. Lancet 2004;364(9431):369-379. DOI: 10.1016/S0140-6736(04)16727-5.

12. Koehler P, Tacke D, Cornely OA. Aspergillosis of bones and joints-a review from 2002 until today. Mycoses 2014;57(6):323-335. DOI: 10.1111/myc.12165.

13. Haidar R, Der Boghossian A, Atiyeh B. Duration of post-surgical antibiotics in chronic osteomyelitis: empiric or evidence-based? Int J Infect Dis 2010;14(9):e752-e758. DOI: 10.1016/j.jijid.2010.01.005.

14. Brady RA, Leid JG, Calhoun JH, et al. Osteomyelitis and the role of biofilms in chronic infection. Pathog Dis 2008;52(1):13-22. DOI: 10.1111/j.1574-695X.2007.00357.x.

15. Sealy PI, Nguyen C, Tucci M, et al. Delivery of antifungal agents using bioactive and nonbioactive bone cements. Ann Pharmacother 2009;43(10):1606-1615. DOI: 10.1345/aph.1M143.

16. Grimsrud C, Raven R, Fothergill A, et al. The in vitro elution characteristics of antifungal-loaded PMMA bone cement and calcium sulfate bone substitute. Orthopedics 2011;34(8):e378-e381. DOI: 10.3928/01477447-20110627-05.

17. Rouse MS, Heijink A, Steckelberg JM, et al. Are anidulafungin or voriconazole released from polymethylmethacrylate in vitro? Clin Orthopaed Relat Res 2011;469(5):1466-1469. DOI: 10.1007/s11999010-1643-7.

18. Wafa H, Grimer RJ, Reddy K, et al. Retrospective evaluation of the incidence of early periprosthetic infection with silver-treated endoprostheses in high-risk patients. Bone Joint J 2015;97(2):252-257. DOI: 10.1302/0301-620X.97B2.34554.

19. Hardes J, Von Eiff C, Streitbuerger A, et al. Reduction of periprosthetic infection with silver-coated megaprostheses in patients with bone sarcoma. J Surg Oncol 2010;101(5):389-395. DOI: 10.1002/jso.21498.

20. Alt V, Bechert $T$, Steinrücke $P$, et al. An in vitro assessment of the antibacterial properties and cytotoxicity of nanoparticulate silver bone cement. Biomaterials 2004;25(18):4383-4391. DOI: 10.1016/ j.biomaterials.2003.10.078.

21. Panáček $A$, Kolář $M$, Večeřová $R$, et al. Antifungal activity of silver nanoparticles against Candida spp. Biomaterials 2009;30(31): 6333-6340. DOI: 10.1016/j.biomaterials.2009.07.065.

22. Ogar A, Tylko G, Turnau K. Antifungal properties of silver nanoparticles against indoor mould growth. Sci Total Environm 2015;521:305-314. DOI: 10.1016/j.scitotenv.2015.03.101.

23. Khalilpour $\mathrm{P}$, Lampe $\mathrm{K}$, Wagener $\mathrm{M}$, et al. $\mathrm{Ag} / \mathrm{SiO}_{x} \mathrm{C}_{y}$ plasma polymer coating for antimicrobial protection of fracture fixation devices. J Biomed Mater Res Part B Appl Biomater 2010;94(1):196-202.

24. Glehr M, Leithner A, Friesenbichler J, et al. Argyria following the use of silver-coated megaprostheses: no association between the development of local argyria and elevated silver levels. Bone Joint J 2013;95(7):988-992. DOI: 10.1302/0301-620X.95B7.31124.

25. Zhang C, Chen M, Wang G, et al. Pd@ ag Nanosheets in combination with amphotericin B exert a Potent anti-Cryptococcal Fungicidal effect. PLoS ONE 2016;11(6):e0157000. DOI: 10.1371/journal. pone. 0157000 . 